\title{
Inflammatory Periimplant Diseases and the Periodontal Connection Question
}

\author{
Claudio Passariello ${ }^{1}$ Dario Di Nardo² Luca Testarelli² \\ ${ }^{1}$ Department of Public Health and Infectious Diseases, Sapienza \\ University of Rome, Rome, Italy \\ 2Department of Oral and Maxillofacial Sciences, Sapienza University \\ Address for correspondence Dario Di Nardo, DDS, PhD. \\ Department of Oral and Maxillofacial Sciences, Sapienza University \\ of Rome, Rome, Italy (e-mail: dario.dinardo@uniroma1.it).
} of Rome, Rome, Italy

Eur J Dent 2019;13:119-123

\begin{abstract}
Implant therapy has become a widespread reality in modern dentistry. Nevertheless, dental implants can fail due to different causes, among which inflammatory peri-implant diseases (IPDs) are a major challenge, with prevalences that are much higher than previously believed.

Specific searches were undertaken for each question raised between October and November 2017, in the PubMed website database (US National Library of Medicine, National Institutes of Health; Bethesda, Maryland, United States). Only articles written in English and published from 2007 onward were considered initially. The following keywords were used in the searches "periimplantitis (PI)," "periimplant mucositis (PM)," "dental implant failure," "periimplant microbiota," "periodontal microbiota," "implant failure" (no temporal limit), and "foreign body reaction" (no temporal limit). The selection process resulted in the selection of 239 articles that were analyzed in

Keywords

- gingivitis

- pathogenesis

- periimplant mucositis

- periimplantitis

- periodontitis detail in elaborating this review. The reference list was limited to the 47 most relevant articles due to editorial limits of this Journal.

Intrinsic differences between natural teeth and dental implants are able to give rise to inflammatory diseases that share only minor and scarcely relevant characters, and would consequently deserve different and specifically designed instruments and strategies, for both diagnosis and therapy.
\end{abstract}

\section{Introduction}

Pilot studies on osseointegration and pioneer experiences on dental implantology opened the way to the possibility to restore missing teeth, with high success rates, by means of dental implants. ${ }^{1-3}$

As for any other kind of implanted artificial device,,5 dental implants can undergo failure, the major challenge being inflammatory periimplant diseases (IPD).

IPDs have been long regarded as manifestations of periodontal diseases affecting dental implants, ${ }^{6}$ in spite of significant differences existing between the periodontium and the intimate implant to alveolar bone connection. ${ }^{7}$

A dental implant is considered as failed when it is not/ no longer functionally adequate; consequently, an implant affected by IPD is not considered as failed till periimplant bone resorption (or loss of bone to implant contact) has evolved to make the implant no more functionally valid.

Although a dental implant can fail due to several mechanical factors like fracture of the implant and/or of the abutment that could make an implant to be inadequate for the anchorage of a prosthesis; the major clinical challenge are correlated with failures caused by biological factors, as the inhibition of the process of osseointegration (early failures) or the loss of a previously achieved osseointegration (late failures). ${ }^{8}$

\section{Methods}

The present review aims to analyze relevant scientific data on the periimplant/periodontal question and to give an answer to openly debated arguments concerning biologic 
implant failures. Critical aspects of the subject are pointed out as questions being raised in the paper.

Specific searches were undertaken for each question raised between October and November 2017, in the PubMed website database (US National Library of Medicine, National Institutes of Health; Bethesda, Maryland, United States). Only articles written in English and published from 2007 onward were considered initially. The following keywords were used in the searches "periimplantitis (PI)," "periimplant mucositis (PM)," "dental implant failure," "periimplant microbiota," "periodontal microbiota," "implant failure" (no temporal limit), and "foreign body reaction" (no temporal limit).

Both review articles and research articles considered pertinent and relevant for discussion were selected and analyzed in detail. All reference lists of the selected articles were then hand-searched for additional papers that were considered relevant. The selection process resulted in the selection of 239 articles that were analyzed in detail in elaborating this review. The reference list was limited to the 47 most relevant articles due to editorial limits of this Journal.

\section{What Can Cause a Biologic Failure?}

Understanding of nature, causes, and pathogenic mechanisms implicated in biologic failures is essential for prevention and adequate clinical management.

More than two decades ago, a study performed on 30 dental implants surgically removed for primary failure in the absence of clinical evidence of infection suggested that primary failures could be correlated to the bacterial colonization of implant surfaces, ${ }^{9}$ although cases of the first kind are frequently ascribed to poor surgery, lack of primary stability, or overheating. ${ }^{10}$

Two main causes of biological implant failure have been extensively investigated in the past: functional overload and infection.

Functional overload was once considered able to cause periimplant bone resorption ${ }^{11}$; at present, most authors consider that the role of overload, in the absence of inflammation, has been largely overestimated. ${ }^{12,13}$ As opposite, the role of periimplant infection is well demonstrated. ${ }^{6,14,15}$

\section{Is There a Link between IPD and Periodontal Diseases?}

Two distinct forms of IPD have been recognized, PM, and PI. ${ }^{6}$ PM is defined as a pathological process whose main clinical characteristic is bleeding on gentle probing and in which erythema, swelling, and/or suppuration may also be present, while PI is defined as a plaque-associated pathological condition occurring in tissues around dental implants, characterized by inflammation in the periimplant mucosa and subsequent progressive loss of supporting bone. ${ }^{16} \mathrm{PM}$ is a pathology occurring at very high frequencies (about $50 \%$ of implanted sites), while the prevalence of PI is reported with significant variations in different studies. ${ }^{14,17}$

The similarities existing, respectively, between gingivitis and PM and between periodontitis and PI are of immediate evidence, but this does not imply that PM and gingivitis, on one side, and PI and periodontitis, on the other side, are effectively fraternal diseases.

According to some authoritative researchers, marginal bone loss around implants should be regarded as a complication to treatment, initiated by inadequate clinical handling, use of inadequate implants, and improper selection of patients, while infection is a secondary phenomenon. ${ }^{10,18}$ According to them, osseointegration is a "balanced foreign body reaction" that, when perturbed by a complicating event, gives rise to marginal bone loss, while infectious events initiate only later. ${ }^{10}$

The assumption that host-implant interactions are comparable to a foreign body reaction embedding implants in bone, just as it happens with Bio-Oss particles used for sinus floor augmentation ${ }^{18,19}$ is questionable: at the titanium-bone interface, a direct contact of bone and titanium, and a bone ingrowth in the nanostructured surface oxide, is detected ${ }^{20,21}$; quite differently, a layer rich in multinucleated giant cells, typically suggesting a foreign body reaction, ${ }^{22}$ is observed to encapsulate Bio-Oss particles. ${ }^{19}$

A pathological process resembling a foreign body reaction was described in cases of aseptic loosening of implants used in orthopaedics, ${ }^{23}$ as a consequence of the presence of wear particles liberated from the bearing surface of implants. ${ }^{24}$ Although enhanced titanium concentrations were documented also in tissues surrounding some types of dental implants, no clinical evidence of periimplant disease was detected in these cases. ${ }^{25}$

Overall, IPD should be considered as bacterially driven inflammatory processes. As to the possibility to make a parallel between them and periodontal diseases, ${ }^{6,15}$ it seems reasonable to consider that the question could be different for the two pairs of pathologies.

\section{Are Gingivitis and Periimplant Mucositis Fraternal Diseases?}

Just like gingivitis, PM is an inflammatory disease restricted to the periimplant mucosa of an implant in function. The primary and main cause of gingivitis is an excessive accumulation of bacteria. ${ }^{26}$ The relevant microbiological aspect of gingivitis is the amount of bacterial biofilm growing next to the gingival margin, not the type of bacteria. ${ }^{27}$

Both gingivitis and PM are highly prevalent, ${ }^{14,26}$ fully reversible diseases, ${ }^{6,28}$ and are mostly associated with changes in the amount of bacterial biofilm. As for gingivitis PM can be experimentally induced in humans by subgingival ligatures..$^{29}$ Clinical parameters, inflammatory markers, and microbiome data obtained from naturally and implanted sites during a period of oral hygiene abstention substantially confirm that gingivitis and PM share many clinical, inflammatory and microbiological traits, although implants accumulate less plaque and undergo more heterogeneous structural shifts at the microbiome level. ${ }^{30}$ Moreover, PM shows a suspected tendency to progress to PI easier and earlier than gingivitis to periodontitis. ${ }^{15}$ 
It can be consequently concluded that, although with minor differences, gingivitis and PM are fraternal diseases.

\section{Are Periodontitis and Periimplantitis Fraternal Diseases?}

It has long been considered reasonable that periodontitis and PI are fraternal diseases, but although the two diseases share a number of common traits, they also show relevant distinctive characters.

In reviewing the scientific literature on the histopathological characteristics of the two diseases, Berglundh et $\mathrm{al}^{31}$ concluded that periodontitis and PI are characterized by critical histopathological differences.

Differently from ligature-induced periodontitis, ligatureinduced PI is not self-limiting when ligatures are removed. ${ }^{32,33}$

In recent years, concepts on the etiology and pathogenesis of periodontitis have been changing and these changes deserve detailed consideration to understand if periodontitis and PI are to be considered similar.

Periodontitis is an inflammatory disease characterized by a complex etiopathogenesis, not constantly related to oral hygiene. The extreme complexity of the oral and dental microbiota has made the efforts to define the etiology of the different clinical forms of periodontitis difficult.

The introduction of molecular methods of analysis allowed researchers to analyze and compare large number of samples and to understand that most of the species that were once regarded as putative periodontal pathogens were also detected from healthy sites, in evident contrast with their status of putative pathogens. ${ }^{34,35}$ These observations put in evidence that healthy controls host a periodontal microbiota dominated by Gram-positive facultative species in a state of dynamic equilibrium with defensive mechanisms of the host; when this equilibrium is broken as a consequence of excessive plaque accumulation or host/environmental factors that reduce the efficiency of defensive mechanisms, the consequence is inflammation and tissue breakdown (i.e., periodontitis).

In such an essentially dysbiotic condition, the chemical and biochemical composition of the microenvironment changes toward a greater abundance of peptides and a relative reduction in polysaccharides, playing a prominent role in promoting the microbial shift toward a Gram-negative anaerobic microbiota. ${ }^{36}$

Similar modifications have been described also as the cause of the inflammatory bowel diseases in which gut dysbiosis and severe inflammation are induced by an imbalanced diet (excess protein intake), genetic predisposition, or stress. ${ }^{37}$

The dysbiotic hypothesis is able to explain the presence of low numbers of putative periodontal pathogens also in healthy controls and their significant numeric increase in periodontal lesions. The dysbiotic nature of periodontitis opens the way to innovative therapeutic approaches, following experiences extensively investigated in the field of inflammatory bowel diseases. ${ }^{38}$

Those microbial taxa that we have learned to indicate as putative periodontal pathogens are the necessary participants to a banquet that is prepared by dysfunctional defensive mechanisms of the host, but alone they are not sufficient to start and maintain the pathologic process. ${ }^{39}$

The view of periodontitis as a multifactorial inflammatory disease associated with a dysbiosis at the periodontal biofilm level has relevant implications for the question whether periodontitis and PI are comparable diseases or not.

Till recently most authors agreed that the biofilm from periodontal pockets contributes to bacterial colonization of implant surfaces in partially edentulous patients and that periodontitis is a factor of risk for PI. ${ }^{40}$ Recently, however, several studies have been suggesting that periodontitis and PI could differ significantly also at the microbiological level.

The presence of the so-called uncommon periodontal pathogens (namely Staphylococcus aureus, Pseudomonas aeruginosa, enterococci, and several Gram-negative enteric rods) is extremely frequent in $\mathrm{PI}^{41}$ Although studies reporting on the detection of common periodontal pathogens from PI affected sites are numerous, they are biased by conceptual limitations: in fact, they promote the paradigm that the periodontal and periimplant habitats are similar and consequently adopt molecular methods targeted at relevant microbial members of the periodontal microbiota. ${ }^{42}$

The periodontal environment is significantly different from the composite assembly of different materials that characterizes the implant to abutment connection area. ${ }^{43}$

Cultural methods contributed to demonstrate that periimplant biofilms are different from periodontal ones: they allowed understanding that staphylococci, enterococci, $P$. aeruginosa, enteric bacteria, and some yeasts are found as frequently as periodontal bacteria at implanted sites. ${ }^{44}$

The application of cultivation-independent open-ended molecular approaches based on 16S-rDNA sequencing and pyrosequencing demonstrated that while the periodontitisassociated communities possess greater diversity and richness as compared to those associated with healthy teeth, the PI-associated communities are characterized by the lowest values of diversity and the fewest number of species among the four studied categories. ${ }^{45,46}$ This finding is strongly suggestive of the hypothesis that PI is a simple, although microbially heterogeneous infection and this would make it substantially different from periodontitis.

Dabdoub et $\mathrm{al}^{46}$ applied the pyrosequencing approach to identify the degree of congruence between 81 adjacent periimplant and periodontal microbiomes in both health and disease conditions: no individual was shown to share more than one-third of the resident microbiota between tooth and implant, and only a minority of dental implants adjacent to periodontal sites positive for putative pathogens included in the red complex resulted in turn positive for the same microorganisms. Far more important, the most abundant species in the two environments were constantly distinct.

These observations overall suggest that the hypothesis identifying the periodontal microbiota as a microbial reservoir for implant colonization and disease lacks any scientific evidence.

A comparative transcriptome analysis on tissue specimens from PI and periodontitis-affected sites revealed that PI and periodontitis share only minimal features, strongly 
suggesting that they are distinct diseases in which substantially different biological mechanisms are involved. ${ }^{47}$

\section{Discussion and Conclusions}

Since its heroic first steps, implant dentistry has been paralleled to periodontology. IPDs were clinically distinguished in PM and PI, making an evident and direct parallel with the periodontal counterparts gingivitis and periodontitis.

Data from biological studies are introducing a significant change in our view on the relationships existing between the periodontium and its diseases on one side and dental implants and their diseases on the other side.

Available experimental data suggest that, even if significant microbiological differences are evident, it could be reasonable to make a correlation between gingivitis and PM, since both diseases are mainly triggered by inadequate oral hygiene, are characterized by inflammation limited to the soft tissues immediately surrounding the emergence of the root/implant, and both promptly respond to enhanced oral hygiene and heal without any evident permanent damage.

On the other hand, periodontitis and PI cannot be considered as fraternal disease. In fact, they are different with regard to etiology, pathogenesis, patterns of progression, and response to treatment.

Etiologically, according to the results of recent researches, they are characterized by oppositely directed evolutionary patterns of the resident microbiota (enhanced diversity in periodontitis as compared to a significant simplification in PI). With regard to pathogenesis, the two conditions not only differ in the composition of cellular infiltrates and in the extension of lesions but also in the pattern of progression.

Although natural teeth and dental implants are inserted within similar anatomic and environmental contexts, their intrinsic differences are able to give rise to inflammatory diseases that share only minor and scarcely relevant characters, and would consequently deserve different and specifically designed instruments and strategies (both for diagnosis and therapy). Once the periodontal paradigm is broken, several convictions that have been based on it, should be revised, as for the one considering periodontal pockets as a reservoir of infection for PI. Periodontitis is certainly a factor of risk for PI, but the fulcrum around which this risk move is not to be found in the microbial inhabitants of periodontal pockets, but rather in the conditions that allowed the periodontal lesion to develop.

Further research is still necessary to better understand why and how an implant becomes infected, to design more appropriate and accurate diagnostic tools to allow early diagnosis and to better address therapy.

\section{Financial Support and Sponsorship}

Nil.

\section{Conflicts of Interest}

None declared.

\section{References}

1 Buser D, Sennerby L, De Bruyn H. Modern implant dentistry based on osseointegration: 50 years of progress, current trends and open questions. Periodontol 2000 2017;73(1):7-21

2 Chappuis V, Buser R, Brägger U, Bornstein MM, Salvi GE, Buser D. Long-term outcomes of dental implants with a titanium plasma-sprayed surface: a 20-year prospective case series study in partially edentulous patients. Clin Implant Dent Relat Res 2013;15(6):780-790

3 Vandeweghe S, Ferreira D, Vermeersch L, Mariën M, De Bruyn $\mathrm{H}$. Long-term retrospective follow-up of turned and moderately rough implants in the edentulous jaw. Clin Oral Implants Res 2016;27(4):421-426

4 Lamagni T. Epidemiology and burden of prosthetic joint infections. J Antimicrob Chemother 2014;69(Suppl 1) :i5-i10

5 Inui T, Bandyk DF. Vascular surgical site infection: risk factors and preventive measures. Semin Vasc Surg 2015;28(3-4):201-207

6 Lindhe J, Meyle J; Group D of European Workshop on Periodontology. Peri-implant diseases: Consensus Report of the Sixth European Workshop on Periodontology. J Clin Periodontol 2008;35(8, Suppl):282-285

7 Heitz-Mayfield LJ, Lang NP. Comparative biology of chronic and aggressive periodontitis vs. peri-implantitis. Periodontol 2000 2010;53:167-181

8 Chrcanovic BR, Kisch J, Albrektsson T, Wennerberg A. Factors influencing early dental implant failures. J Dent Res 2016;95(9):995-1002

9 Passariello C, Berlutti F, Selan L, et al. Microbiological and morphological analysis of dental implants removed for incomplete osseointegration. Microb Ecol Health Dis 1993;6(4):203-207

10 Chrcanovic BR, Albrektsson T, Wennerberg A. Reasons for failures of oral implants. J Oral Rehabil 2014;41(6):443-476

11 Rangert B, Krogh PH, Langer B, Van Roekel N. Bending overload and implant fracture: a retrospective clinical analysis. Int J Oral Maxillofac Implants 1995;10(3):326-334

12 Chang M, Chronopoulos V, Mattheos N. Impact of excessive occlusal load on successfully-osseointegrated dental implants: a literature review. J Investig Clin Dent 2013;4(3):142-150

13 Duyck J, Vandamme K. The effect of loading on peri-implant bone: a critical review of the literature. J Oral Rehabil 2014;41(10):783-794

14 Zitzmann NU, Berglundh T. Definition and prevalence of peri-implant diseases. J Clin Periodontol 2008;35(8, Suppl): 286-291

15 Lang NP, Berglundh T; Working Group 4 of Seventh European Workshop on Periodontology. Periimplant diseases: where are we now?--Consensus of the Seventh European Workshop on Periodontology. J Clin Periodontol 2011;38(Suppl 11):178-181

16 Berglundh T, Armitage G, Araujo MG, et al. Peri-implant diseases and conditions: Consensus report of workgroup 4 of the 2017 world workshop on the classification of periodontal and peri-implant diseases and conditions. J Periodontol 2018;89(Suppl 1):S313-S318

17 Koldsland OC, Scheie AA, Aass AM. Prevalence of periimplantitis related to severity of the disease with different degrees of bone loss. J Periodontol 2010;81(2):231-238

18 Albrektsson T, Dahlin C, Jemt T, Sennerby L, Turri A, Wennerberg A. Is marginal bone loss around oral implants the result of a provoked foreign body reaction? Clin Implant Dent Relat Res 2014;16(2):155-165

19 Mordenfeld A, Hallman M, Johansson CB, Albrektsson T. Histological and histomorphometrical analyses of biopsies harvested 11 years after maxillary sinus floor augmentation with deproteinized bovine and autogenous bone. Clin Oral Implants Res 2010;21(9):961-970 
20 Palmquist A, Omar OM, Esposito M, Lausmaa J, Thomsen P. Titanium oral implants: surface characteristics, interface biology and clinical outcome. J R Soc Interface 2010;7Suppl 5): S515-S527

21 Palmquist A, Grandfield K, Norlindh B, Mattsson T, Brånemark $\mathrm{R}$, Thomsen $\mathrm{P}$. Bone-titanium oxide interface in humans revealed by transmission electron microscopy and electron tomography. J R Soc Interface 2012;9(67):396-400

22 Anderson JM, Rodriguez A, Chang DT. Foreign body reaction to biomaterials. Semin Immunol 2008;20(2):86-100

23 Cavalli L, Brandi ML. Periprosthetic bone loss: diagnostic and therapeutic approaches. F1000 Res 2014;2:266

24 Dyskova T, Gallo J, Kriegova E. The role of the chemokine system in tissue response to prosthetic by-products leading to periprosthetic osteolysis and aseptic loosening. Front Immunol 2017;8:1026

25 Tawse-Smith A, Ma S, Duncan WJ, Gray A, Reid MR, Rich AM. Implications of wear at the titanium-zirconia implant-abutment interface on the health of peri-implant tissues. Int J Oral Maxillofac Implants 2017;32(3):599-609

26 Page RC, Kornman KS. The pathogenesis of human periodontitis: an introduction. Periodontol 2000 1997;14:9-11

27 Konstantinidis IK, Kotsakis GA, Gerdes S, Walter MH. Cross-sectional study on the prevalence and risk indicators of peri-implant diseases. Eur J Oral Implantology 2015;8(1):75-88

28 Ata-Ali J, Ata-Ali F, Galindo-Moreno P. Treatment of periimplant mucositis: a systematic review of randomized controlled trials. Implant Dent 2015;24(1):13-18

29 Pontoriero R, Tonelli MP, Carnevale G, Mombelli A, Nyman SR, Lang NP. Experimentally induced peri-implant mucositis. A clinical study in humans. Clin Oral Implants Res 1994;5(4):254-259

30 Schincaglia GP, Hong BY, Rosania A, et al. Clinical, immune, and microbiome traits of gingivitis and peri-implant mucositis. J Dent Res 2017;96(1):47-55

31 Berglundh T, Zitzmann NU, Donati M. Are peri-implantitis lesions different from periodontitis lesions? J Clin Periodontol 2011;38(Suppl 11):188-202

32 Lindhe J, Berglundh T, Ericsson I, Liljenberg B, Marinello C. Experimental breakdown of peri-implant and periodontal tissues. A study in the beagle dog. Clin Oral Implants Res 1992;3(1):9-16

33 Albouy JP, Abrahamsson I, Persson LG, Berglundh T. Spontaneous progression of ligatured induced peri-implantitis at implants with different surface characteristics. An experimental study in dogs II: histological observations. Clin Oral Implants Res 2009;20(4):366-371
34 Papapanou PN. Population studies of microbial ecology in periodontal health and disease. Ann Periodontol 2002;7(1):54-61

35 Avila M, Ojcius DM, Yilmaz O. The oral microbiota: living with a permanent guest. DNA Cell Biol 2009;28(8):405-411

36 Lamont RJ, Hajishengallis G. Polymicrobial synergy and dysbiosis in inflammatory disease. Trends Mol Med 2015;21(3):172-183

37 Marchesi JR, Adams DH, Fava F, et al. The gut microbiota and host health: a new clinical frontier. Gut 2016;65(2):330-339

38 Liévin-Le Moal V, Servin AL. Anti-infective activities of lactobacillus strains in the human intestinal microbiota: from probiotics to gastrointestinal anti-infectious biotherapeutic agents. Clin Microbiol Rev 2014;27(2):167-199

39 Feng Z, Weinberg A. Role of bacteria in health and disease of periodontal tissues. Periodontol 2000 2006;40:50-76

40 Mombelli A, Décaillet F. The characteristics of biofilms in peri-implant disease. J Clin Periodontol 2011;38(Suppl 11) :203-213

41 Charalampakis G, Leonhardt Å, Rabe P, Dahlén G. Clinical and microbiological characteristics of peri-implantitis cases: a retrospective multicentre study. Clin Oral Implants Res 2012;23(9):1045-1054

42 Robitaille N, Reed DN, Walters JD, Kumar PS. Periodontal and peri-implant diseases: identical or fraternal infections? Mol Oral Microbiol 2016;31(4):285-301

43 Perera-Costa D, Bruque JM, González-Martín ML, GómezGarcía AC, Vadillo-Rodríguez V. Studying the influence of surface topography on bacterial adhesion using spatially organized microtopographic surface patterns. Langmuir 2014;30(16):4633-4641

44 Lafaurie GI, Sabogal MA, Castillo DM, et al. Microbiome and microbial biofilm profiles of peri-implantitis: a systematic review. J Periodontol 2017;88(10):1066-1089

45 Kumar PS, Mason MR, Brooker MR, O'Brien K. Pyrosequencing reveals unique microbial signatures associated with healthy and failing dental implants. J Clin Periodontol 2012;39(5):425-433

46 Dabdoub SM, Tsigarida AA, Kumar PS. Patient-specific analysis of periodontal and peri-implant microbiomes. J Dent Res 2013;92(12, Suppl):168S-175S

47 Becker ST, Beck-Broichsitter BE, Graetz C, Dörfer CE, Wiltfang J, Häsler R. Peri-implantitis versus periodontitis: functional differences indicated by transcriptome profiling. Clin Implant Dent Relat Res 2014;16(3):401-411 